\title{
Dynamic Cellular Manufacturing to Simplify Scheduling in Cell Based Production
} Systems

\section{VR KANNAN}

James Madison University, Harrisonburg, VA, USA

\section{GHOSH}

Georgia Institute of Technology, Atlanta, Ga, USA

\begin{abstract}
Previous research has shown that the formation of temporary, logical manufacturing cells in a job shop, overcomes problems of high setup frequency typically found in job shops. In addition, this form of cellular configuration is more flexible than tranditional, group technology based cellular configurations. This research demonstrates that an added benefit of a dynamic cellular configuration is that it simplifies production scheduling, eliminating the need for more complex dispatching rules. In particular, it eliminates the need for further setup reduction when scheduling parts from the same family. Improvements in performance when employing setup reduction are shown to occur only when the premium associated with intra-family setup reduction is very high, and are attributable to reductions in flow time variance.
\end{abstract}

Key words--cellular manufacturing, group technology, scheduling, simulation

\section{INTRODUCTION}

ALTHOUGH BATCH producers continue to implement cellular manufacturing (CM) systems to improve production efficiency, some with reported success [23], it is still unclear how effective such systems are. Several studies question reports that converting job shops using process layouts to cellular layouts can yield improvements in throughput and due date performance $[4,12,22]$, concerns centering around the validity of the comparisons made. Controlled comparisons suggest that few environments are conducive to successful CM implementations $[4,13,20]$ unless $\mathrm{CM}$ is implemented in a manner that would be hard to replicate in a job shop [19-21]. Primary concerns regarding the suitability of CM for small batch production are that it is inflexible to changes in demand patterns and to shifts in workload patterns.

An alternative way to achieve the setup efficiencies of CM systems without compromis-ing flexibility is to view cells not as permanent, physical structures, but as temporary, virtual structures. Cells can be formed by using scheduling mechanisms to temporarily allocate machines in a job shop to part families [7]. Cells are allowed to evolve and dissolve on a dynamic, real time basis, enabling them to respond more effectively to changes in workload and to shifts in the locations of bottlenecks. This way the setup efficiencies of cellular shops are achieved while simultaneously exploiting the flexibility inherent in a job shop. This paper shows that additional benefits of this form of cellular configuration are that shop floor control is simplified, there is a diminished need for further setup reduction, and that decisions regarding the prioritization of individual jobs are made largely insignificant.

Routing parts outside their designated cells to reduce imbalances in utilization does offer the potential for a cellular configuration to out-perform a process layout [20]. This however runs counter to the objective of a cellular layout to simplify material handling and reduce setup frequency. 
The conversion of a process layout to a cellular layout should yield several benefits. These include lower flow times and reduced work in process inventory [5]. Though some empirical evidence bears this out [23], its validity has been questioned on the grounds that the new and existing systems are typically dissimilar enough to make direct comparison meaningless [4, 12, 22]. The time that elapses between operating the two systems allows changes in the environment to take place, further compromising the validity of comparisons. In contrast, comparisons made under more controlled conditions using simulation models consistently suggest that cellular layouts perform well only under limited conditions. Specifically, they perform well only when setup and material handling times are very high and demand patterns are stable [13]. Permanent machine dedication even in a process layout significantly hampers routing flexibility, resulting in the build up of queues and thus poor flow time performance [4]. These results have been verified analytically [20]. If processing efficiencies are obtained in a cellular configuration, e.g. setup times and lot sizes reduced [20], processing times reduced [21], or jobs split into smaller transfer batches to facilitate simultaneous processing on multiple machines [19], the configuration can be made to outperform an otherwise similar job shop. However, this adds to the problem of comparing dissimilar systems. In addition, these improvements still fail to address the inability of CM to respond effectively to changes in demand patterns.

Attempts to improve CM performance have also been made using shop floor control procedures though with limited success. The use of group scheduling rules that recognize sequence dependencies improves performance [16], though not to the extent that performance is comparable to that of a process layout [3].

\section{DYNAMIC CELLULAR MANUFACTURING}

In a dynamic cellular manufacturing (DCM) configuration, machines are allocated to part families rather than individual jobs, as is the case in a traditional cellular configuration. Unlike a traditional cellular configuration, however, this allocation is neither permanent, nor does it involve physical re-organization of an existing job shop. Idle machines are assigned to families that have an immediate need to use them, with priority going to those families which do not currently have access to a machine of the type in question. As long as batches of parts from this family reside in the current queue, they have priority over other batches in dispatching decisions. Once the machine is no longer needed by the family or the family has access to multiples of the machine type, the machine is relinquished and made available to other families that require its use but lack access to a machine of this type. If no such families exist, the machine remains allocated to the current family. As machines from different process departments are allocated to families, family specific routings develop through the shop similar to those found in traditional manufacturing cells. However, these routings can evolve over time in response to changes in demand patterns and machine availability. The result is a mechanism to promote the formation and dissolution of manufacturing cells based on an efficient allocation of machine resources to part families. DCM thus represents an alternative to traditional cellular systems. It is therefore a potential manufacturing configuration for industries as diverse as machine tools, medical equipment and office furniture, as well as others where production is carried out in a batch mode.

An earlier study [7] showed that this configuration yields better flow time, work in process and due date performance than traditional cellular or job shops over a range of conditions. A fundamental characteristic of DCM illustrated by the study is its reduction of the frequency of major (inter-family) setups similar to that found in traditional cellular systems. The study did not however indicate whether further setup reduction once a machine has been allocated to a family is of additional benefit. In other words, is there any advantage to be gained from 
scheduling batches using rules employing the repetitive lots (RL) or similar logic [6]. These require that all batches of the same part in a queue be processed in sequence prior to a setup change. Such a situation can arise if the queue contains different orders for the same part or if batches are split into smaller transfer batches in order to improve material flow. This logic yielded significant performance improvements in traditional job shop [6] and cellular environments [3]. If further setup reduction improves performance in a shop employing DCM, one should seek to implement scheduling schemes that facilitate this. However, if it is of limited or no benefit, this eliminates the need to use more complex scheduling rules instead of simpler, traditional dispatching rules. This in turn reduces the potential for batches to be delayed if a large number of batches requiring the current shop have priority.

This study will investigate whether intra-family setup reduction significantly affects the performance of DCM systems and if so, under what conditions. This will be accomplished by simulating the performance of DCM under a variety of conditions where there are different premiums associated with this type of setup reduction. The experimental design includes five experimental factors. Two of these, DCM implementation and dispatching rule, define scheduling conditions within the shop. The remaining factors, major setup time ratio, minor setup time ratio, and batch size, are factors that can be expected to affect the impact of intrafamily setup reduction.

\section{EXPERIMENTAL FACTORS}

\section{DCM implementation}

Idle machines are allocated to part families using one of two heuristics. Machines are allocated either to the family with the lowest average slack per batch in the current queue (FAMS), or to the family requiring the fewest additional machines to complete a cell (MINM) that meets the processing needs of all family members. While FAMS considers only batches in the current queue when machine allocation decisions are made, MINM considers production activity in all other departments, allowing it to make a more conscious, active attempt to form cells. These heuristics performed well in the earlier study [7].

\section{Dispatching rule}

Four dispatching rules are used to sequence batches of parts from the same part family. These consist of two traditional dispatching rules that have been shown to perform well in both job and cellular shops $[1,14]$, shortest processing time (SPT), and minimum slack (SLK), as well as the repetitive lots counterparts of these rules (SPT-RL, SLK-RL). By including these variants of the dispatching rules, the impact of intra-family setup reduction can be ascertained.

\section{Major setup time}

The performance of DCM systems has been shown to depend on the magnitude of major setup time [7]. To capture this effect, two levels of setup time/run time (S/R) ratio are examined. At the low level, major setup time is $11.33 \mathrm{~min}$, and at the high level it is $22.66 \mathrm{~min}$. These correspond to $\mathrm{S} / \mathrm{R}$ ratios of $1 / 3$ and $2 / 3$ respectively [9] and are typical of those found in practice [23].

\section{Minor setup time}

The benefit of intra-family setup reduction can be expected to depend in part on how long these setups take since this defines how much productive capacity is sacrificed when a minor 
setup is incurred. Two levels of the ratio of minor to major setup time (M/M) are examined to address this issue. At the low level, minor setup time is one-quarter of the major setup time [4], and at the high level it is one-half of the major setup time [13]. These ratios are again similar to those observed in industry [23].

\section{Batch size}

It is reasonable to expect that the benefits of intra-family setup reduction will depend on how long batches must wait if others must be processed ahead of them. This will vary with changes in batch size. Three batch sizes are examined in this study. The default batch size is 100 [7]. In addition, batch sizes of 50 and 200 are considered [6].

For comparative purposes, the shop is also observed when operated as a traditional job shop (JS). In other words, at each process department batches are sequenced solely according to processing time or slack with no regard to family affiliation. To more accurately represent the operation of an actual job shop and to compensate for the fact that as described, it employs no setup reduction, batches of the same part are processed in sequence prior to invoking the SPT or SLK dispatching rules.

\section{SIMULATION ENVIRONMENT AND PERFORMANCE MEASURES}

A simulation method of a hypothetical, 30 machine job shop similar to [13] was used to carry out the study. The shop consists of eight process departments, each containing three or four identical machines. Forty distinct parts belonging to one of five different part families are produced. Orders arrive according to a Poisson process with the mean inter-arrival rate established to yield average shop utilization of $80 \%$ when family allocation is based on the MINM heuristic and SPT dispatching is used. Orders are for a single batch of one part. Each part requires between two and six operations with no more than one in a given department. Operation processing times are normally distributed with a mean of 34.33 min and a standard deviation of $0.25 \mathrm{~min}$ for a batch of size 100. Due dates are established using the total work content method with an allowance of three times the estimated total processing time [1]. Forklift trucks are used to transport parts at a rate of $5 \mathrm{mph}$. Loading and unloading times are uniformly distributed in the interval $(1,5)$.

Three performance measures are observed, the mean and standard deviation of flow time, and mean tardiness. These allow insights to be gained into the impact of setup reduction on both flow time and due date performance. Simulation run conditions were established using pilot runs to identify the initialization period [18], and batch size to ensure independence [8] and normality of batch means [2]. Common random numbers were used for all input processes but one to reduce variance while maintaining independence [11]. The simulation model was written using the SIMAN simulation language [15] and user written FORTRAN sub-routines.

\section{RESULTS}

Treatment means for the three performance measures are presented in Tables 1-3. Analyses of variance indicated that for each performance measure, all main and most interaction effects are significant ( $\mathrm{ct}=0.05$ ). To illustrate the behavior of the scheduling mechanisms under the different setup time and batch size conditions examined, Tukey multiple comparisons of treatment means were carried out separately for each batch size, of each combination of shop configuration and dispatching rule for all combinations of setup time condition (Tables 4-6). Treatments grouped together are those with statistically insignificant differences in mean performance. 
Regardless of batch size and setup time conditions, the DCM configurations clearly yield better mean flow time performance than a traditional job shop environment (Table 4). This is consistent with the results of the previous study [7]. The one exception is when batch size is 200 and S/R and M/M ratios are 1/3 and 1/2 respectively. Under these conditions, though the job shop yields the highest mean flow times, performance is statistically indistinguishable from that of the DCM implementations. More importantly however, there is little to be gained from the use of repetitive lots based scheduling in a DCM environment. Even when conditions are most conducive to setup reduction using RL (high setup times, small batch sizes), its use typically yields only marginally lower flow times, but any differences are not statistically significant. The only such occasion when the use of RL based scheduling does yield a significant improvement over the corresponding non-RL dispatching rule is when the FAMS/SPT implementation is used (Fig. 1). Under other setup time and batch size scenarios, RL scheduling again yields small, statistically insignificant differences in performance.

The results for the standard deviation of flow time are largely similar to those for mean flow time (Table 5). Except when batch size is 100 or greater, the job shop yields the poorest performance. The use of RL scheduling yields lower, but in general not statistically lower flow time variance. However, when the SPT dispatching rule is used, the use of RL does yield significantly lower flow time variance when using DCM. This occurs for all batch sizes when $\mathrm{S} / \mathrm{R}$ and $\mathrm{M} / \mathrm{M}$ ratios are $1 / 3$ and $1 / 2$ respectively and the MINM/SPT implementation is used, and when the FAMS/SPT or MINM/SPT implementations are used with both S/R and M/M ratios at their high levels (Fig. 2). By reducing intra-family setup frequency, RL scheduling reduces the impact of a major source of flow time variance. With RL scheduling, total batch processing time at a machine (operation processing time + intra-family setup time) for most batches consists solely of the operation processing time. When RL scheduling is not used, identical batches may on occasion be processed in sequence without the need for an additional intra-family setup. However, in many cases, an additional setup will be required. 
Table I. Treatment means for mean flow time

\begin{tabular}{|c|c|c|c|c|c|c|c|c|c|c|c|c|c|}
\hline \multirow{3}{*}{$\begin{array}{l}\text { Batch sier } \\
\text { S/R } \\
\mathrm{M} / \mathrm{M}\end{array}$} & & \multicolumn{4}{|c|}{50} & \multicolumn{4}{|c|}{100} & \multicolumn{4}{|c|}{200} \\
\hline & & \multicolumn{2}{|c|}{ Low } & \multicolumn{2}{|c|}{ High } & \multicolumn{2}{|c|}{ Low } & \multicolumn{2}{|c|}{ High } & \multicolumn{2}{|c|}{ Low } & \multicolumn{2}{|c|}{ High } \\
\hline & & Low & High & Low & High & Low & High & Low & High & Low & High & Low & High \\
\hline Shop & \multicolumn{13}{|l|}{ Disp. rule } \\
\hline \multirow[t]{4}{*}{ FAMS } & SPT & 124.76 & 130.20 & 158.33 & 173.19 & 210.16 & 215.83 & 245.27 & 258.23 & 389.59 & 396.87 & 415.71 & 430.72 \\
\hline & SLK & 124.45 & 129.85 & 158.17 & 170.66 & 209.81 & 216.55 & 244.08 & 256.12 & 383.15 & 396.70 & 415.19 & 427.70 \\
\hline & SPT-RL. & 123.49 & 127.73 & 156.96 & 167.14 & 208.73 & 212.94 & 242.42 & 250.88 & 386.80 & 392.43 & 412.62 & 422.97 \\
\hline & SLK-RL & 123.54 & 127.60 & 157.38 & 165.47 & 208.24 & 213.57 & 242.88 & 261.49 & 385.90 & 39189 & 412.01 & 420.95 \\
\hline \multirow[t]{4}{*}{ MINM } & SPT & 127.74 & 132.31 & 162.96 & 175.54 & 213.55 & 218.39 & 250.49 & 260.57 & 393.62 & 400,98 & 422.33 & 435.04 \\
\hline & SLK & 126.78 & 132.28 & 162.85 & 174.83 & 212.91 & 219.92 & 249.84 & 260.90 & 392.80 & 399.33 & 421.34 & 433.64 \\
\hline & SPT-RL & 126.37 & 129.80 & 160.93 & 170.06 & 211.58 & 215.76 & 24832 & 254.72 & 391.91 & 395.15 & 419.66 & 427,84 \\
\hline & SLK-RL & 126.17 & 129.56 & 160.87 & 170.81 & 211.43 & 215.98 & 247.42 & 255.58 & 390.56 & 394.90 & 418.63 & 425.89 \\
\hline \multirow[t]{2}{*}{ IS } & $\mathrm{SPT}$ & 144.54 & 140.89 & 192.05 & 191.22 & 234.26 & 227.83 & 286.24 & 275.93 & 419.88 & 413.53 & 462.89 & 454.16 \\
\hline & SLK & 145.59 & 141.11 & 192.60 & 191.66 & 233.19 & 229.52 & 285.28 & 278.93 & 420.53 & 414.51 & 461.86 & 45204 \\
\hline
\end{tabular}

Table 2. Treatment means for standard deviation of flow time

\begin{tabular}{|c|c|c|c|c|c|c|c|c|c|c|c|c|c|}
\hline \multirow{3}{*}{$\begin{array}{l}\text { Butch size } \\
\text { S/R } \\
M / M\end{array}$} & & \multicolumn{4}{|c|}{50} & \multicolumn{4}{|c|}{100} & \multicolumn{4}{|c|}{200} \\
\hline & & \multicolumn{2}{|c|}{ Low } & \multicolumn{2}{|c|}{ High } & \multicolumn{2}{|c|}{ Low } & \multicolumn{2}{|c|}{ High } & \multicolumn{2}{|c|}{ Low } & \multicolumn{2}{|c|}{ High } \\
\hline & & Low & High & Low & $\mathrm{High}$ & Low & High & Low & High & Low & High & Low & High \\
\hline Shop & Disp, fule & & & & & & & & & & & & \\
\hline FAMS & $\begin{array}{l}\text { SPT } \\
\text { SLK } \\
\text { SPT-RL } \\
\text { SLK.RL. }\end{array}$ & $\begin{array}{l}52.90 \\
50.14 \\
50.03 \\
49.20\end{array}$ & $\begin{array}{l}56.56 \\
52.73 \\
51.92 \\
50.45\end{array}$ & $\begin{array}{l}65.14 \\
62.15 \\
63.39 \\
61.50\end{array}$ & $\begin{array}{l}75,42 \\
67.09 \\
66,94 \\
64.69\end{array}$ & $\begin{array}{l}94.91 \\
88.98 \\
88.19 \\
86.74\end{array}$ & $\begin{array}{l}99.13 \\
93.46 \\
91.17 \\
89.76\end{array}$ & $\begin{array}{r}105.65 \\
99.98 \\
99.81 \\
98.99\end{array}$ & $\begin{array}{l}116.43 \\
106.38 \\
102.77 \\
101.54\end{array}$ & $\begin{array}{l}192.84 \\
172.76 \\
177.30 \\
169.03\end{array}$ & $\begin{array}{l}201.78 \\
177.98 \\
182.07 \\
172.66\end{array}$ & $\begin{array}{l}188.54 \\
177.13 \\
176.93 \\
172.64\end{array}$ & $\begin{array}{l}199.85 \\
183.65 \\
182.02 \\
176.15\end{array}$ \\
\hline MINM & $\begin{array}{l}\text { SPT } \\
\text { SLK } \\
\text { SPT-RL } \\
\text { SLK-RL. }\end{array}$ & $\begin{array}{l}57.10 \\
52.17 \\
53.92 \\
51.62\end{array}$ & $\begin{array}{l}60.64 \\
54.72 \\
55.16 \\
52.24\end{array}$ & $\begin{array}{l}70.84 \\
65.69 \\
67.01 \\
64.60\end{array}$ & $\begin{array}{l}79.85 \\
70.49 \\
70.85 \\
67.60\end{array}$ & $\begin{array}{r}100.75 \\
92.26 \\
93.10 \\
90.02\end{array}$ & $\begin{array}{r}103.25 \\
97.59 \\
96.04 \\
92.67\end{array}$ & $\begin{array}{l}113.08 \\
105.42 \\
108.12 \\
102.51\end{array}$ & $\begin{array}{l}120.85 \\
110.87 \\
108.24 \\
105.76\end{array}$ & $\begin{array}{l}202.13 \\
177.99 \\
186.98 \\
175.00\end{array}$ & $\begin{array}{l}210.57 \\
182.53 \\
186.11 \\
176.60\end{array}$ & $\begin{array}{l}199.58 \\
183.13 \\
186.59 \\
179.16\end{array}$ & $\begin{array}{l}213.88 \\
190.24 \\
192.16 \\
181.69\end{array}$ \\
\hline IS & $\begin{array}{l}\text { SPT } \\
\text { SLK } \\
\end{array}$ & $\begin{array}{l}n .7 \\
n 0.29 \\
\end{array}$ & $\begin{array}{l}68.05 \\
65.78 \\
\end{array}$ & $\begin{array}{r}98.82 \\
89.29 \\
\end{array}$ & $\begin{array}{l}92.43 \\
86.11 \\
\end{array}$ & $\begin{array}{l}122.51 \\
116.49\end{array}$ & $\begin{array}{l}112.39 \\
112.58\end{array}$ & $\begin{array}{l}150.57 \\
138.88\end{array}$ & $\begin{array}{l}134.43 \\
132,46\end{array}$ & $\begin{array}{l}221.75 \\
216.00\end{array}$ & $\begin{array}{l}212.01 \\
210.03\end{array}$ & $\begin{array}{l}242.28 \\
232.19\end{array}$ & $\begin{array}{l}225.56 \\
222.76\end{array}$ \\
\hline
\end{tabular}

Table 3. Trtatment means for mean tardiness

\begin{tabular}{|c|c|c|c|c|c|c|c|c|c|c|c|c|c|}
\hline \multirow{3}{*}{$\begin{array}{l}\text { Batch size } \\
\text { S/R } \\
\text { M/M }\end{array}$} & \multirow{3}{*}{ xe } & \multicolumn{4}{|c|}{50} & \multicolumn{4}{|c|}{100} & \multicolumn{4}{|c|}{200} \\
\hline & & \multicolumn{2}{|c|}{ Low } & \multicolumn{2}{|c|}{ High } & \multicolumn{2}{|c|}{ Low } & \multicolumn{2}{|c|}{ High } & \multicolumn{2}{|c|}{ Low } & \multicolumn{2}{|c|}{ High } \\
\hline & & Low & High & Low & High & Low & High & Low & High & Low & High & Low & High \\
\hline Shop & Disp. rule & & & & & & & & & & & & \\
\hline FAMS & $\begin{array}{l}\text { SPT } \\
\text { SLK } \\
\text { SPT-RL } \\
\text { SLK-RL }\end{array}$ & $\begin{array}{l}2.18 \\
0.29 \\
1.39 \\
0.33\end{array}$ & $\begin{array}{l}3.19 \\
0.71 \\
1.92 \\
0.48\end{array}$ & $\begin{array}{l}5.36 \\
2.63 \\
4.47 \\
2.61\end{array}$ & $\begin{array}{r}11.12 \\
6.27 \\
7.01 \\
4.86\end{array}$ & $\begin{array}{l}2.90 \\
0.17 \\
1.87 \\
0.24\end{array}$ & $\begin{array}{l}3.42 \\
0.34 \\
2.00 \\
0.34\end{array}$ & $\begin{array}{l}3.89 \\
0.46 \\
2.58 \\
0.57\end{array}$ & $\begin{array}{l}6.63 \\
1.31 \\
3.09 \\
0.97\end{array}$ & $\begin{array}{l}7.66 \\
0.22 \\
4.77 \\
0.56\end{array}$ & $\begin{array}{l}9.40 \\
0.44 \\
5.44 \\
0.67\end{array}$ & $\begin{array}{l}5.92 \\
0.29 \\
3.56 \\
0.35\end{array}$ & $\begin{array}{l}8.17 \\
0.67 \\
4.60 \\
0.65\end{array}$ \\
\hline MINM & $\begin{array}{l}\text { SPT } \\
\text { SLK } \\
\text { SPT-RL } \\
\text { SLK-RL }\end{array}$ & $\begin{array}{l}3.08 \\
0.52 \\
2.17 \\
0.56\end{array}$ & $\begin{array}{l}4.24 \\
1.18 \\
2.65 \\
0.77\end{array}$ & $\begin{array}{l}7.55 \\
4.33 \\
6.09 \\
3.88\end{array}$ & $\begin{array}{r}12.58 \\
8.59 \\
8.74 \\
6.58\end{array}$ & $\begin{array}{l}4.10 \\
0.39 \\
2.376 \\
0.46\end{array}$ & $\begin{array}{l}4.05 \\
0.76 \\
2.70 \\
0.64\end{array}$ & $\begin{array}{l}5.66 \\
1.06 \\
3.97 \\
1.01\end{array}$ & $\begin{array}{l}7.21 \\
2.36 \\
4.09 \\
1.73\end{array}$ & $\begin{array}{l}8.92 \\
0.50 \\
5.84 \\
0.93\end{array}$ & $\begin{array}{r}10.47 \\
0.80 \\
5.86 \\
1.10\end{array}$ & $\begin{array}{l}7.64 \\
0.46 \\
4.85 \\
0.80\end{array}$ & $\begin{array}{r}10.22 \\
1.20 \\
6.10 \\
1.03\end{array}$ \\
\hline JS & $\begin{array}{l}\text { SPT } \\
\text { SLK }\end{array}$ & $\begin{array}{l}9.47 \\
3.71 \\
\end{array}$ & $\begin{array}{l}7.33 \\
3.35 \\
\end{array}$ & $\begin{array}{l}25.81 \\
21.19 \\
\end{array}$ & $\begin{array}{l}22.95 \\
19.12\end{array}$ & $\begin{array}{l}9.71 \\
1.33 \\
\end{array}$ & $\begin{array}{l}6.39 \\
0.77\end{array}$ & $\begin{array}{r}19.26 \\
6.25\end{array}$ & $\begin{array}{r}12.36 \\
4.47\end{array}$ & $\begin{array}{r}14.17 \\
1.43\end{array}$ & $\begin{array}{r}12.13 \\
0.82\end{array}$ & $\begin{array}{r}17.75 \\
2.26\end{array}$ & $\begin{array}{r}14.49 \\
1.37\end{array}$ \\
\hline
\end{tabular}


Table 4. Tukey multiple comparisons for mean fow time

\begin{tabular}{|c|c|c|c|c|c|c|}
\hline $\begin{array}{l}\text { Batch siat } \\
\text { Selup time } \\
\text { conditions }\end{array}$ & 50 & & 100 & & 200 & \\
\hline $\begin{array}{l}S / R=1 / 3 \\
M / M=1 / 4\end{array}$ & $\begin{array}{l}\text { FAMS/SPT-RL } \\
\text { FAMS/SLK-RL. } \\
\text { FAMS/SLK } \\
\text { FAMS/SPT } \\
\text { MINM/SLK-RL } \\
\text { MINM/SPT-RL } \\
\text { MINM/SLK } \\
\text { MINM/SPT } \\
\text { JS/SPT } \\
\text { JS/SL.K }\end{array}$ & i & $\begin{array}{l}\text { FAMS/SLKK.RL } \\
\text { FAMS/SPT-RL } \\
\text { FAMS/SLKK } \\
\text { FAMS/SPT } \\
\text { MINM/SL.K-RL } \\
\text { MINM/SPT-RL } \\
\text { MINM/SL.K } \\
\text { MINM/SPT } \\
\text { JS/SLK } \\
\text { JS/SPT }\end{array}$ & $\begin{array}{l}i \\
i \\
i \\
i \\
i \\
i \\
i \\
i\end{array}$ & $\begin{array}{l}\text { FAMS/SLKK-RL } \\
\text { FAMS/SPT-RL } \\
\text { FAMS/SLK } \\
\text { FAMS/SPT } \\
\text { MINM/SLK-RL } \\
\text { MINM/SPT-RL } \\
\text { MINM/SLK } \\
\text { MINM/SPT } \\
\text { JS/SPT } \\
\text { JS/SLK }\end{array}$ & i \\
\hline $\begin{array}{l}S / R=1 / 3 \\
M / M=1 / 2\end{array}$ & $\begin{array}{l}\text { FAMS/SLK-RL } \\
\text { FAMS/SPT-RL } \\
\text { MINM/SLK-RL } \\
\text { MINM/SPT-RL } \\
\text { FAMS/SLK } \\
\text { FAMS/SPT } \\
\text { MINM/SLK } \\
\text { MINM/SPT } \\
\text { IS/SPT } \\
\text { IS/SL.K }\end{array}$ & i & $\begin{array}{l}\text { FAMS/SPT-RL } \\
\text { FAMS/SLK-RL } \\
\text { MINM/SPT-RL } \\
\text { FAMS/SPT } \\
\text { MINM/SLK-RL. } \\
\text { FAMS/SLK } \\
\text { MINM/SPT } \\
\text { MINM/SL.K } \\
\text { JS/SPT } \\
\text { JS/SLK }\end{array}$ & $\begin{array}{l}1 \\
i \\
i \\
i \\
i \\
i \\
i \\
i \\
i \\
i \\
i\end{array}$ & $\begin{array}{l}\text { FAMS/SLK-RL } \\
\text { FAMS/SPT-RL } \\
\text { MINM/SLK-RL } \\
\text { MINM/SPT-RL } \\
\text { FAMS/SLKK } \\
\text { FAMS/SPT } \\
\text { MINM/SLK } \\
\text { MINM/SPT } \\
\text { JS/SPT } \\
\text { JS/SLK }\end{array}$ & $i$ \\
\hline $\begin{array}{l}S / R=2 / 3 \\
M / M=1,4\end{array}$ & $\begin{array}{l}\text { FAMS/SPT-RL } \\
\text { FAMS/SLK-RL } \\
\text { FAMS/SLK } \\
\text { FAMS/SPT } \\
\text { MINM/SLK-RL } \\
\text { MINM/SPT-RL } \\
\text { MINM/SLK } \\
\text { MINM/SPT } \\
\text { JS/SPT } \\
\text { JS/SLK }\end{array}$ & 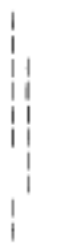 & $\begin{array}{l}\text { FAMS/SPT-RL } \\
\text { FAMS/SLKK-RL } \\
\text { FAMS/SLK } \\
\text { FAMS/SPT } \\
\text { MINM/SLK-RL } \\
\text { MINM/SPT-RL } \\
\text { MINM/SL.K } \\
\text { MINM/SPT } \\
\text { JS/SLK } \\
\text { JS/SPT }\end{array}$ & $\begin{array}{l}i \\
i \\
i \\
i \\
i \\
i \\
i\end{array}$ & $\begin{array}{l}\text { FAMS/SLK-RL } \\
\text { FAMS/SPT-RL } \\
\text { FAMS/SLK } \\
\text { FAMS/SPT } \\
\text { MINM/SLK-RL. } \\
\text { MINM/SPT-RL } \\
\text { MINM/SLK } \\
\text { MINM/SPT } \\
\text { JS/SLKK } \\
\text { JS/SPT }\end{array}$ & $\begin{array}{l}1 \\
i \\
i \\
i \\
i \\
i \\
i\end{array}$ \\
\hline $\begin{array}{l}S / R=2 / 3 \\
M / M=1 / 2\end{array}$ & $\begin{array}{l}\text { FAMS/SPT-RL } \\
\text { FAMS/SLK-RL } \\
\text { MINM/SLK-RL } \\
\text { FAMS/SLKK } \\
\text { MINM/SPT-RL } \\
\text { FAMS/SPT } \\
\text { MINM/SLK } \\
\text { MINM/SPT } \\
\text { IS/SPT } \\
\text { IS/SLK }\end{array}$ & $\begin{array}{lll}1 & \\
1 & 1 \\
1 & 1 & 1 \\
1 & 1 \\
1 & 1 \\
1 & 1 \\
1 & 1 \\
1 & 1\end{array}$ & $\begin{array}{l}\text { FAMS/SPT-RL } \\
\text { FAMS/SLK-RL } \\
\text { MINM/SPT-RL } \\
\text { MINM/SLK-RL } \\
\text { FAMS/SLKK } \\
\text { FAMS/SPT } \\
\text { MINM/SPT } \\
\text { MINM/SLK } \\
\text { JS/SPT } \\
\text { JS/SLK }\end{array}$ & $\begin{array}{l}1 \\
1 \\
11 \\
11 \\
11 \\
11 \\
1 \\
1 \\
1\end{array}$ & $\begin{array}{l}\text { FAMS/SLK-RL } \\
\text { FAMS/SPT-RL } \\
\text { MINM/SLK-RL } \\
\text { FAMS/SLK } \\
\text { MINM/SPT-RL } \\
\text { FAMS/SPT } \\
\text { MINM/SLK } \\
\text { MINM/SPT } \\
\text { JS/SLK } \\
\text { JS/SPT }\end{array}$ & $\begin{array}{l}1 \\
i \\
i \\
i \\
i \\
i \\
i \\
i \\
i\end{array}$ \\
\hline
\end{tabular}

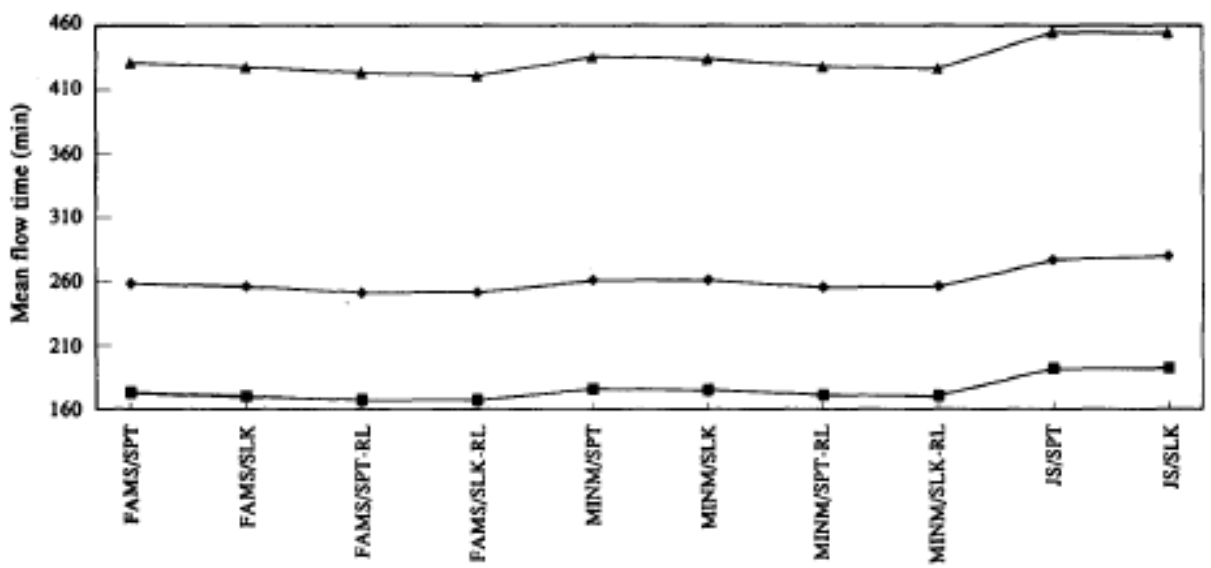

Shop configuration/scheduling mechanism

Fig. 1. Mean flow time performance $(S / R=2 / 3, M / M=1 / 2)$. $\mathbf{D}$. Batch size $=50 ; \bullet$. batch size $-100 ; \Delta$, batch size -200 . 
Table 5. Tukey multiple comparisons for the slandard deviation of flow time

\begin{tabular}{|c|c|c|c|c|c|c|}
\hline $\begin{array}{l}\text { Batch size } \\
\text { Seiup time } \\
\text { conditions }\end{array}$ & 50 & & 100 & & 200 & \\
\hline $\begin{array}{l}\mathrm{S} / \mathrm{R}=1 / 3 \\
\mathrm{M} / \mathrm{M}=1 / 4\end{array}$ & $\begin{array}{l}\text { FAMS/SLK-RL. } \\
\text { FAMS/SPT-RL. } \\
\text { FAMS/SLK } \\
\text { MINM/SLK-RL } \\
\text { MINM/SLLK } \\
\text { FAMS/SPT } \\
\text { MINM/SPT-RL. } \\
\text { MINM/SPT } \\
\text { JS/SLK } \\
\text { JS/SPT }\end{array}$ & $\begin{array}{l}1 \\
1 \\
1 \\
i \\
i \\
i\end{array}$ & $\begin{array}{l}\text { FAMS/SLKK } \\
\text { FAMS/SLK-RL } \\
\text { MINM/SLKK } \\
\text { MINM/SL-K-RL } \\
\text { JS/SLLK } \\
\text { FAMS/SPT-RL } \\
\text { MINM/SPT-RL } \\
\text { FAMS/SPT } \\
\text { MINM/SPT } \\
\text { JS/SPT }\end{array}$ & 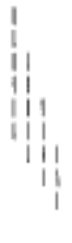 & $\begin{array}{l}\text { FAMS/SLK } \\
\text { MINM/SLK } \\
\text { FAMS/SLK-RL } \\
\text { MINM/SLK-RL. } \\
\text { JS/SL.K } \\
\text { FAMS/SPT-RL } \\
\text { MINM/SPT-RL } \\
\text { FAMS/SPT } \\
\text { MINM/SPT } \\
\text { IS/SPT }\end{array}$ & 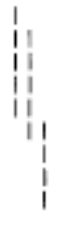 \\
\hline $\begin{array}{l}S_{j} R=1 / 3 \\
M / M=1 / 2\end{array}$ & $\begin{array}{l}\text { FAMS/SLK-RL } \\
\text { FAMS/SPT-RL } \\
\text { MINM/SLK-RL } \\
\text { FAMS/SL.K } \\
\text { MINM/SLK } \\
\text { MINM/SPT-RL. } \\
\text { FAMS/SPT } \\
\text { MINM/SPT } \\
\text { JS/SLK } \\
\text { JS/SPT }\end{array}$ & ii & $\begin{array}{l}\text { FAMS/SLK-RL } \\
\text { FAMS/SLK } \\
\text { MINM/SLK-RL } \\
\text { MINM/SLK } \\
\text { JS/SLK } \\
\text { FAMS/SPT-RL. } \\
\text { MINM/SPT-RL } \\
\text { FAMS/SPT } \\
\text { MINM/SPT } \\
\text { JS/SPT }\end{array}$ & $\begin{array}{ll}1 \\
11 \\
11 \\
11 \\
11 \\
11 \\
1\end{array}$ & $\begin{array}{l}\text { FAMS/SL.K } \\
\text { FAMS/SL.K-RL. } \\
\text { MINM/SLK } \\
\text { JS-SLK } \\
\text { MINM/SLK-RL } \\
\text { FAMS/SPT-RL. } \\
\text { MINM/SPT-RL. } \\
\text { FAMS/SPT } \\
\text { MINM/SPT } \\
\text { IS/SPT }\end{array}$ & $i_{i}^{i}$ \\
\hline $\begin{array}{l}\mathrm{S} / \mathrm{R}=2 / 3 \\
\mathrm{M} / \mathrm{M}=1 / 6\end{array}$ & $\begin{array}{l}\text { FAMS/SL.K-RL } \\
\text { FAMS/SLKK } \\
\text { FAMS/SPT-RL } \\
\text { MINM/SLK-RL } \\
\text { FAMS/SPT } \\
\text { MINM/SLK } \\
\text { MINM/SPT-RL } \\
\text { MINM/SPT } \\
\text { JS/SLK } \\
\text { JS/SPT }\end{array}$ & $\begin{array}{l}1 \\
1 \\
11 \\
11 \\
11 \\
11 \\
11\end{array}$ & $\begin{array}{l}\text { FAMS/SLK } \\
\text { FAMS/SLK-RL } \\
\text { MINM/SLK-RL } \\
\text { MINM/SLK } \\
\text { FAMS/SPT-RL } \\
\text { FAMS/SPT } \\
\text { MINM/SPT-RL } \\
\text { MINM/SPT } \\
\text { IS/SLK } \\
\text { JS/SPT }\end{array}$ & ii & $\begin{array}{l}\text { FAMS/SL.K } \\
\text { FAMS/SLK-RL } \\
\text { MINM/SLK } \\
\text { MINM/SLK-RL } \\
\text { JS/SLK } \\
\text { FAMS/SPT-RL } \\
\text { MINM/SPT-RL } \\
\text { FAMS/SPT } \\
\text { MINM/SPT } \\
\text { JS/SPT }\end{array}$ & $\begin{array}{lll}1 & & \\
1 & & \\
1 & & \\
1 & & \\
1 & 1 & \\
1 & 1 & 1 \\
1 & 1 & 1 \\
1 & 1 & 1 \\
1 & 1\end{array}$ \\
\hline $\begin{array}{l}\mathrm{S} / \mathrm{R}=2 / 3 \\
\mathrm{M} / \mathrm{M}=1 / 2\end{array}$ & $\begin{array}{l}\text { FAMS/SLK-RL } \\
\text { FAMS/SPT-RL } \\
\text { FAMS/SL.K } \\
\text { MINM/SLK-RL } \\
\text { MINM/SLK } \\
\text { MINM/SPT-RL } \\
\text { FAMS/SPT } \\
\text { MINM/SPT } \\
\text { JS/SPT } \\
\text { JS/SLK }\end{array}$ & $\begin{array}{ll}1 & \\
11 \\
i 1 \\
11 \\
11 \\
1 \\
i\end{array}$ & $\begin{array}{l}\text { FAMS/SLKK-RL. } \\
\text { FAMS/SLK } \\
\text { MINM/SLK-RL } \\
\text { MINM/SLK } \\
\text { FAMS/SPT-RL } \\
\text { MINM/SPT-RL } \\
\text { JS/SLK } \\
\text { FAMS/SPT } \\
\text { MINM/SPT } \\
\text { IS/SPT }\end{array}$ & 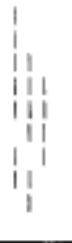 & $\begin{array}{l}\text { FAMS/SLKK-RL. } \\
\text { FAMS/SLKK } \\
\text { MINM/SLK-RL. } \\
\text { MINM/SLK } \\
\text { JS/SLK } \\
\text { FAMS/SPT-RL } \\
\text { MINM/SPT-RL. } \\
\text { FAMS/SPT } \\
\text { MINM/SPT } \\
\text { JS/SPT }\end{array}$ & $\begin{array}{l}1 \\
i \\
i 1 \\
11 \\
11 \\
i \\
i \\
i\end{array}$ \\
\hline
\end{tabular}

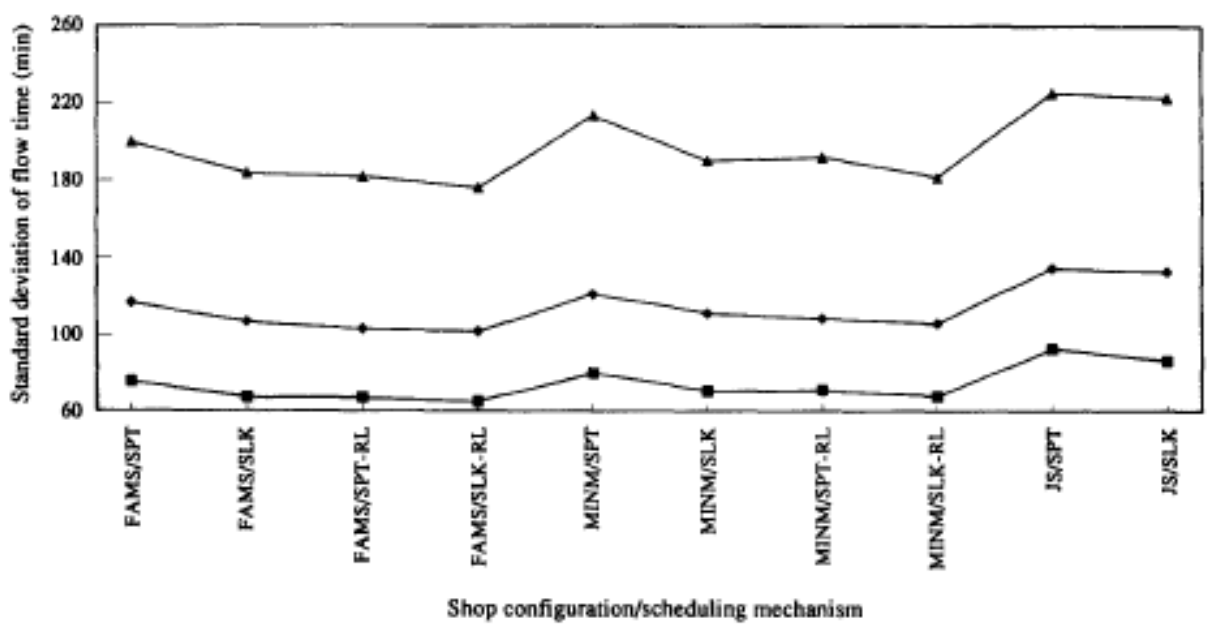

Fig. 2. Standard deviation of flow time performance $(\mathrm{S} / \mathrm{R}=2 / 3 . \mathrm{M} / \mathrm{M}-1 / 2)$. $\mathbf{Q}$. Batch size $=90$ : - batch size $=100 ; \boldsymbol{\Delta}$, batch size $=200$. 


\begin{tabular}{|c|c|c|c|c|c|c|}
\hline \multirow{2}{*}{$\begin{array}{l}\text { Batch size } \\
\text { Setup time } \\
\text { conditions } \\
\mathrm{S} / \mathrm{R}=1 / 3 \\
\mathrm{M} / \mathrm{M}=1 / \mathrm{S}\end{array}$} & \multicolumn{2}{|l|}{50} & \multicolumn{2}{|l|}{100} & \multicolumn{2}{|l|}{200} \\
\hline & $\begin{array}{l}\text { FAMS/SLK } \\
\text { FAMS/SLK-RL } \\
\text { MINM/SLK } \\
\text { MINM/SLK-RL } \\
\text { FAMS/SPT-RL } \\
\text { MINM/SPT-RL } \\
\text { FAMS/SPT } \\
\text { MINM/SPT } \\
\text { JS/SLK } \\
\text { JS/SPT }\end{array}$ & $\begin{array}{lll}1 & \\
1 & 1 \\
1 & 1 \\
1 & 1 \\
1 & 1 & 1 \\
1 & 1 & 1 \\
1 & 1 & 1 \\
1 & 1\end{array}$ & $\begin{array}{l}\text { FAMS/SLK-RL } \\
\text { FAMS/SPT-RL } \\
\text { FAMS/SLK } \\
\text { MINM/SLK-RL } \\
\text { MINM/SLK } \\
\text { MINM/SPT-RL } \\
\text { FAMS/SPT } \\
\text { MINM/SPT } \\
\text { JS/SLK } \\
\text { IS/SPT }\end{array}$ & 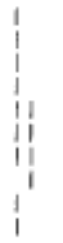 & $\begin{array}{l}\text { FAMS/SL_K-RL } \\
\text { FAMS/SLK } \\
\text { MINM/SLK-RL } \\
\text { FAMS/SPT-RL } \\
\text { MINM/SLK } \\
\text { MINM/SPT-RL } \\
\text { FAMS/SPT } \\
\text { MINM/SPT } \\
\text { JS/SLK } \\
\text { JS/SPT }\end{array}$ & 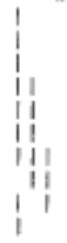 \\
\hline $\begin{array}{l}\mathrm{S} / \mathrm{R}=1 / 3 \\
\mathrm{M} / \mathrm{M}=1 / 2\end{array}$ & $\begin{array}{l}\text { FAMS/SLKK-RL } \\
\text { FAMS/SLK } \\
\text { MINM/SLK-RL } \\
\text { MINM/SLK } \\
\text { FAMS/SPT-RL } \\
\text { JS/SLK } \\
\text { MINM/SPT-RL } \\
\text { FAMS/SPT } \\
\text { MINM/SPT } \\
\text { IS/SPT }\end{array}$ & 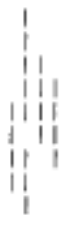 & $\begin{array}{l}\text { FAMS/SLK-RL } \\
\text { FAMS/SPT-RL } \\
\text { MINM/SLK·RL } \\
\text { FAMS/SLK } \\
\text { MINM/SPT.RL } \\
\text { MINM/SLK } \\
\text { FAMS/SPT } \\
\text { MINM/SPT } \\
\text { JS/SPT } \\
\text { JS/SLK }\end{array}$ & i & $\begin{array}{l}\text { FAMS/SL.K-RL } \\
\text { MINM/SLK-RL. } \\
\text { FAMS/SLKK } \\
\text { FAMS/SPT-RL. } \\
\text { MINM/SLK } \\
\text { MINM/SPT-RL. } \\
\text { FAMS/SPT } \\
\text { JS/SLK } \\
\text { MINM/SPT } \\
\text { JS/SPT }\end{array}$ & in \\
\hline $\begin{array}{l}S / R=2 / 3 \\
M / M=1 / 4\end{array}$ & $\begin{array}{l}\text { FAMS/SLK-RL } \\
\text { FAMS/SL.K } \\
\text { MINM/SLK-RL } \\
\text { MINM/SLK } \\
\text { FAMS/SPT-RL } \\
\text { FAMS/SPT } \\
\text { MINM/SPT-RL. } \\
\text { MINM/SPT } \\
\text { JS/SLK } \\
\text { JS/SPT }\end{array}$ & $\begin{array}{ll}1 \\
11 \\
11 \\
11 \\
11\end{array}$ & $\begin{array}{l}\text { FAMS/SLK-RL. } \\
\text { FAMS/SPT-RL. } \\
\text { FAMS/SLK } \\
\text { MINM/SLK-RL } \\
\text { MINM/SLK } \\
\text { FAMS/SPT } \\
\text { MINM/SPT-RL } \\
\text { MINM/SPT } \\
\text { JS/SLK } \\
\text { JS/SPT }\end{array}$ & 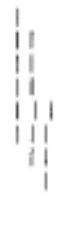 & $\begin{array}{l}\text { FAMS/SLK-RL } \\
\text { FAMS/SPT-RL } \\
\text { FAMS/SLK } \\
\text { MINM/SLK-RL. } \\
\text { MINM/SLK } \\
\text { MINM/SPT-RL } \\
\text { FAMS/SPT } \\
\text { MINM/SPT } \\
\text { JS/SLK } \\
\text { JS/SPT }\end{array}$ & $\begin{array}{l}1 \\
i \\
i \\
i \\
i \\
i \\
i \\
i \\
i\end{array}$ \\
\hline $\begin{array}{l}\mathrm{S} / \mathrm{R}=2 / 3 \\
\mathrm{M} / \mathrm{M}=1 / 2\end{array}$ & $\begin{array}{l}\text { FAMS/SL.K-RL } \\
\text { FAMS/SL.K } \\
\text { MINM/SLK-RL } \\
\text { FAMS/SPT-RL } \\
\text { MINM/SLK } \\
\text { MINM/SPT-RL } \\
\text { FAMS/SPT } \\
\text { MINM/SPT } \\
\text { JS/SLK } \\
\text { JS/SPT }\end{array}$ & $\begin{array}{l}11 \\
11 \\
11 \\
11 \\
11 \\
11\end{array}$ & $\begin{array}{l}\text { FAMS/SLK-RL } \\
\text { FAMS/SPT-RL. } \\
\text { MINM/SLK-RL } \\
\text { FAMS/SLK } \\
\text { MINM/SPT-RL } \\
\text { MINM/SLK } \\
\text { FAMS/SPT } \\
\text { MINM/SPT } \\
\text { JS/SL.K } \\
\text { JS/SPT }\end{array}$ & $\begin{array}{l}1 \\
i \\
i \\
i \\
i \\
i \\
i \\
i\end{array}$ & $\begin{array}{l}\text { FAMS/SLK-RL } \\
\text { MINM/SLK-RL } \\
\text { FAMS/SPT-RL } \\
\text { FAMS/SLK } \\
\text { MINM/SLK } \\
\text { MINM/SPT-RL } \\
\text { FAMS/SPT } \\
\text { MINM/SPT } \\
\text { JS/SLK } \\
\text { JS/SPT }\end{array}$ & $\begin{array}{ll}1 & \\
11 \\
11 \\
11 \\
11 \\
11 \\
1 & 1 \\
1 & 1 \\
1 & 1\end{array}$ \\
\hline
\end{tabular}

The result is an increase in the variance of total batch processing times. This also explains the differences, though insignificant, in mean flow time performance. When SPT dispatching is used, incorporation of the RL logic also serves to prevent discrimination against batches with long operation processing times by increasing their priority if they require the current minor setup. This reduces the impact of another source of variance, thus the greater impact of RL when SPT dispatching is used.

The results for mean tardiness are again similar (Table 6), though with more modest advantages associated with the use of DCM. The use of RL scheduling can improve the due date performance of DCM but only under certain conditions when both S/R and M/M ratios are at their high levels. This can occur when batch size is 50 or 100 and either the FAMS/SPT or MINM/SPT implementation is used, or when batch size is 200 and the MINM/SPT implementation is used (Fig. 3). This can again be attributed to the variance reduction properties of RL described above.

\section{IMPLICATIONS OF DCM FOR BATCH SCHEDULING}

The relatively small impact of intra-family setup reduction in a DCM environment unlike that observed in traditional job shop and cellular environments, suggests that the use of DCM reduces the impact of dispatching decisions. Only when setup times are high does the use of repetitive lots-based scheduling yield any significant advantage over traditional dispatching rules, and here too, only under certain conditions. The use of RL based scheduling is effective in reducing flow time variance which in turn yields improved due date performance. 


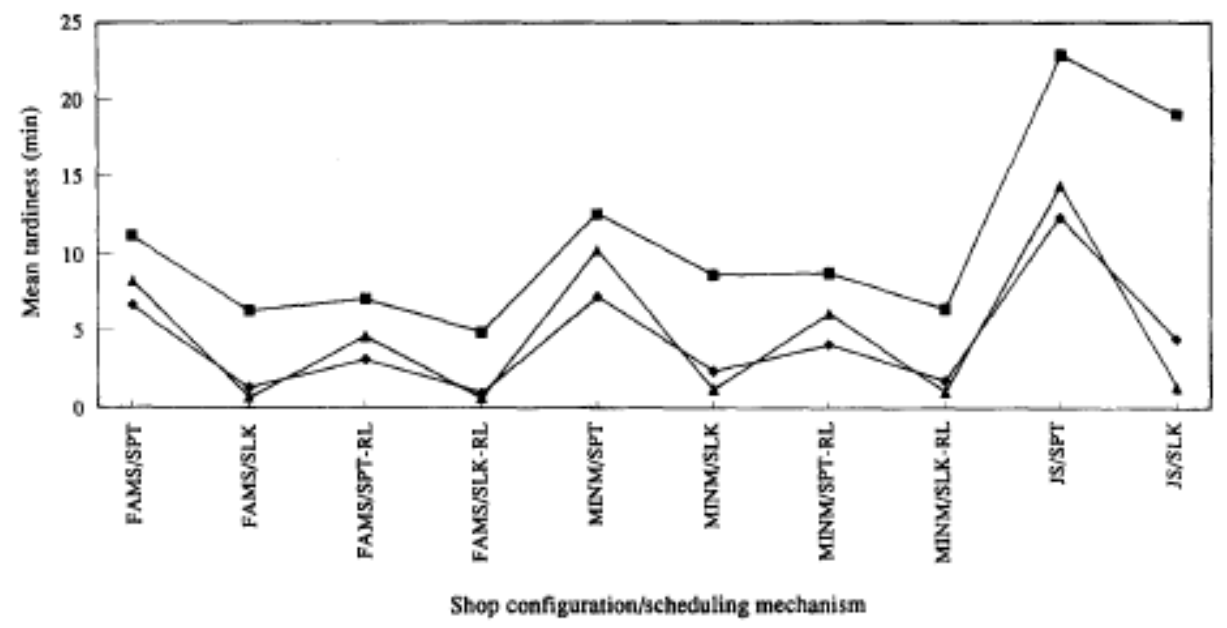

Fig. 3. Mean tardiness performance $(\mathrm{S} / \mathrm{R}=2 / 3, \mathrm{M} / \mathrm{M}-1 / 2)$. D. Batch size $-50 ; \bullet$, batch

size $=100 ; \boldsymbol{\Delta}$. batch size $=200$

However, mean flow time is not improved when minor setup frequency is reduced. Even when the use of RL does reduce flow time variance, it does so under conditions which are inherently vulnerable to high flow time variance, namely when SPT dispatching is used. When SLK dispatching is used, the use of RL does nothing to improve performance. This suggests that the use of RL based scheduling itself adds no value, rather it only acts to reduce the impact of problems that are felt in its absence.

An additional observation is that under those conditions where the use of RL does yield performance improvements, these improvements occur regardless of batch size. As suggested earlier, one might expect intra-family setup reduction to be advantageous when the magnitude of setup time relative to batch processing time is large. However, this appears not to be the case. This suggests either that it is the magnitude of setup time alone that is critical in defining whether the use of RL will be beneficial, or that the ratio of minor setup time to batch processing time must be decreased further (batch size increased) to eliminate the benefits of intra-family setup reduction.

The limited effect of intra-famity setup reduction clearly illustrates the impact DCM has on scheduling decisions. Dispatching schemes are typically used to smooth shop load and manage due dates once jobs have been released to the shop. DCM, by facilitating a more efficient distribution of machine resources, implicitly reduces much of the variance found on the shop floor. D C M acts as a buffer between the order release and shop floor scheduling systems, thereby assuming some of the order release system's responsibility for maintaining an even load and efficient utilization of resources. The result is to eliminate the need for more complex schemes for prioritizing individual jobs similar to the impact of order review and release mechanisms [10]. What is also apparent from the results is that how machines are allocated to families is not as critical as the fact that they are allocated primarily to families and not jobs. This coupled with the fact that whether SPT or SLK job dispatching is used makes little difference implies that instead of machine allocation decisions being made each time a job is completed, decisions can be made less frequently without a significant penalty being incurred. In addition to simplifying the job of the scheduler, this brings added stability to shop floor control activities.

\section{CONCLUSIONS}


With product life cycles shortening and demand patterns becoming more complex, there is an increasing need for manufacturing systems that can respond to a dynamic environment, and for more efficient and effective resource allocation. DCM meets these needs by decreasing the frequency of resource allocation decisions with respect to machine resources, while simultaneously reducing the impact of making poor decisions. From the shop floor manager's perspective, the use of DCM not only decreases the frequency of scheduling decisions, but it reduces the number of variables that must be considered in making those decisions. No longer must he be concerned about which specific jobs are in each queue. Instead he only needs to be concerned about which family a machine is allocated to.

By emphasizing the use of more global shop information in making machine allocation decisions as opposed to more local information, DCM also serves as a complement to the planning system. Though not a substitute for good planning, this does alleviate problems associated with order changes that can affect otherwise good schedules. Such changes can significantly affect the ability to meet promised due dates. Furthermore, in the absence of an adequate planning system, DCM can reduce the instability in shop load and thus erratic shop performance that would occur, without the need for information that is not already available. This allows customer requirements to be met in a more timely, effective manner. Acknowledgement--This work was supported by the James Madison University Program of Grants for Faculty Assistance.

\section{REFERENCES}

1. Baker KR (1984) Sequencing rules and due date assignments in a job shop. Mgmt Sci. 3tl, 1093-1104.

2. Filliben JJ (1975) The probability plot correlation coefficient test for normality. Technometrics 17, I 1 I-I 17.

3. Flynn BB (1987) Repetitive lots: the use of a sequence dependent setup time scheduling procedure in group technology \& traditional shops. J. Opns Mgmt 7, 203-216.

4. Flynn BB and Jacobs FR (1987) An experimental comparison of cellular (group technology) layout with process layout. Decis. Sci. 18, 562-581.

5. Green TJ and Sadowski RP (1984) A review of cellular manufacturing assumptions, advantages and design techniques. J. Opns Mgmt 4, 85-97.

6. Jacobs FR and Bragg DJ (1988) Repetitive lots: flow time reductions through sequencing and dynamic batch sizing. Decis. Sci. 19, 284-291.

7. Kannan VR and Ghosh S (1995) Cellular manufacturing using virtual cells. Int. J. Opns Prod. Mgmt In press.

8. Kleijnen JPC (1987) Statistical Tools For Simulation Practitioners. Marcel Dekker, New York.

9. Mahmoodi F, Tierney EJ and Mosier CT (1992) Dynamic group scheduling heuristics in a flow through cell environment. Decis. Sci. 23, 61-85.

10. Melnyk SA, Ragatz GL and Fredendall LD (1991) Load smoothing by the planning and order review/ release systems: a simulation experiment. J. Opns Mgmt 10, 512-523.

11. Mihram GA (1974) Blocking in simular experimental designs. J. Statist. Comput. Simul. 3, 29-32.

12. Morris JS and Tersine RJ (1989) A comparison of cell loading practices in group technology. J. Manuf. Opns Mgmt 2, 299-313.

13. Morris JS and Tersine RJ (1990) A simulation analysis of factors influencing the attractiveness of group technology cellular layouts. Mgmt Sci. 36, 1567-1578.

14. Mosier CT, Elvers DA and Kelly D (1984) Analysis of group technology scheduling heuristics. Int. J. Prod. Res. 22, 857-875. 
15. Pegden CD (1987) Introduction to SIMAN. Systems Modeling Corp., Sewickley, Pa.

16. Ruben RA, Mosier CT and Mahmoodi F (1993) A comprehensive analysis of group scheduling heuristics in a job shop cell. Int. J. Prod. Res. 31, 1343-1370.

17. Schmeiser BW (1982) Batch size effects in the analysis of simulation output. Opns Res. 311, 556-568.

18. Schruben L, Singh H and Tierney L (1983) Optimal tests for initialization bias in simulation output. Opns Res. 31, 1167-1178.

19. Shafer SM and Charnes JM (1993) Cellular versus functional layouts under a variety of shop operating conditions. Decis. Sci. 24, 665-682.

20. Suresh NC (1992) Partitioning work centres for technology: analytical extension and shop level simulation investigation. Decis. Sci. 23, 267-290.

21. Suresh NC and Meredith JR (1994) Coping with the loss of pooling synergy in cellular manufacturing systems. Mgmt Sci. 411, 466-483.

22. Wemmerlov U and Hyer NL (1987) Research issues in cellular manufacturing. Int. J. Prod. Res. 25, 413-431.

23. Wemmerlov U and Hyer NL (1989) Cellular manufacturing in the US industry: a survey of users. Int. J. Prod. Res. 27, 1511-1530. 